\section{Temperature to heart rate relationship in the neonate}

\author{
NORA HOFER • WILHELM MÜLLER • BERNHARD RESCH
}

Key words: neonate, fever, hypothermia, heart rate, neonatal sepsis

In neonatal intensive care, measurement of heart rate is part of every clinical examination and it is used for monitoring hemodynamic status. However, it is influenced by some exogenous and endogenous factors, such as medication, pain, and stress. (1) Similarly, an increased heart rate is a normal physiological response to fever. Heart rate is known to increase by 10 beats per minute (bpm) per degree centigrade increase in body temperature in children. (2) In order to allow physicians to identify patients who have a higher heart rate than would be expected for a given level of temperature, Thompson et al. (3) created temperature specific heart rate centile charts adaptable to children from three months to ten years. Very few data exist on the relationship of temperature and heart rate in younger infants. The only study on this topic so far was performed in an emergency department that included infants up to the age of 12 months, where they found no linear correlation between fever and heart rate in the group of infants younger than two months. (4) To our knowledge no studies have ever addressed this issue in newborns.

In a retrospective analysis we evaluated the role of fever on heart rate in all neonates hospitalized at our tertiary care teaching neonatal intensive care unit from 2004 to 2007 during the first three days of life. On the ward, body temperature is measured rectally every two to four hours or more frequently when needed. Heart rate is recorded continuously.

Of 863 newborns hospitalized within the first three days of life (32\% term and 68\% preterm), 69 (8\%) had a fever (rectal temperature $>38.5^{\circ} \mathrm{C}$ without evidence for environmental causes including changes in incubator temperature). Body temperature significantly influenced heart rate $(r=.314, p=.009)$ with an increase of $17.5 \mathrm{bpm}$ with each $1^{\circ} \mathrm{C}$ increment in core temperature (95\% confidence interval 4.6-30.4 bpm). In the subgroup of neonates with bacterial sepsis (clinical signs with a positive blood culture and/or laboratory confirmation or a suggestive perinatal history) the increment in heart rate was higher compared to sepsis negative newborns (21.5 bpm (4.9-38.1) vs. 10.5 bpm (-11.7-32.6), respectively, see figure 1). Arterial hypotension was significantly associated with

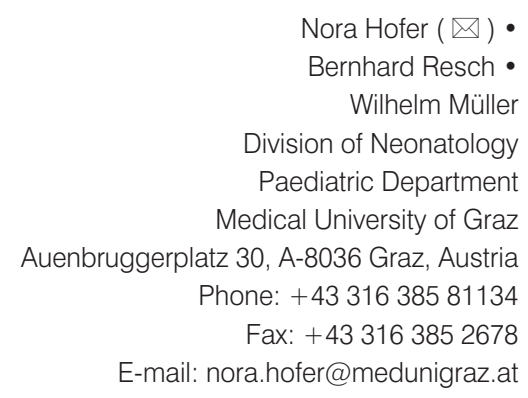

Nora Hofer $(\bowtie) \bullet$ Bernhard Resch • Wilhelm Müller Division of Neonatology Paediatric Department Medical University of Graz Auenbruggerplatz 30, A-8036 Graz, Austria Phone: + 4331638581134

Fax: +43316 3852678 E-mail: nora.hofer@medunigraz.at

an increased heart rate $(p=.018)$. However, the association was not significant when heart rate was adjusted for body temperature. $(p=.067)$

The results of this short analysis demonstrate that the relationship between pulse rate and temperature already applies to neonates. Determining heart rate is part of every clinical examination performed in this age group, and values exceeding the reference range may rapidly lead to the initiation of further diagnostic procedures seeking hemodynamic dysfunction, signs of bacterial infection, congenital or acquired cardiac disorders, etc. The impact of fever and several other factors on heart rate, limit its correct interpretation in the clinical setting. Further research on the impact of fever on heart rate in neonates might better explain the utility of a temperature dependent interpretation of heart rate in this age group.

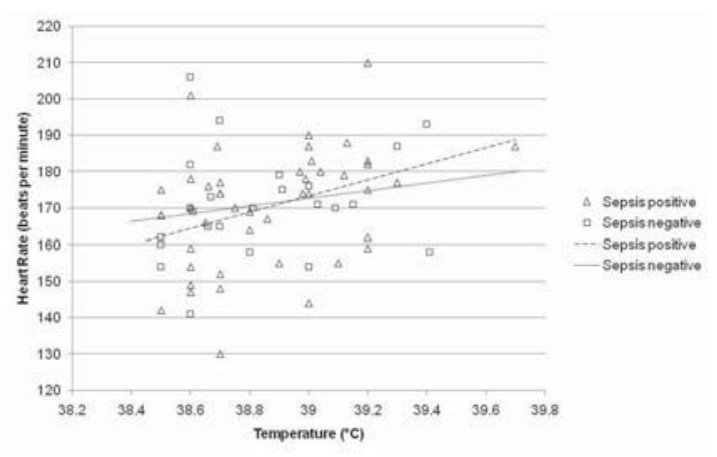

Figure 1. Temperature and pulse of febrile neonates within the first three days of life with and without early onset bacterial sepsis.

\title{
REFERENCES
}

1. de Boode. Clinical monitoring of systemic hemodynamics in critically ill newborns. Early Hum Dev 2010;86:137-41.

2. Davies P, Maconochie I. The relationship between body temperature, heart rate and respiratory rate in children. Emerg Med J 2009;26:641-3.

3. Thompson M, Harnden A, Perera R, Mayon-White R, Smith L, McLeod D, et al. Deriving temperature and age appropriate heart rate centiles for children with acute infections. Arch Dis Child 2009;94:361-5.

4. Hanna CM, Greenes DS. How much tachycardia in infants can be attributed to fever? Ann Emerg Med 2004;43:699-705 